\title{
Aeroacoustics of a Landing Gear Door
}

\author{
Ryu Fattah, ${ }^{*}$ Zhiwei Hu, ${ }^{\dagger}$ and David Angland ${ }^{\ddagger}$ \\ Faculty of Engineering and the Environment \\ University of Southampton, Southampton, Hampshire, SO17 1BJ, UK
}

\begin{abstract}
Numerical and experimental work is presented on the flow around a smooth cylinder and an angled flat plate in a side-by-side configuration, representative of the interaction present in the flow between an aircraft main landing gear leg-door and the main strut. Two parameters are considered for the interaction: the proximity between the two elements, and the leg-door angle relative to the flow. A two-dimensional parametric study, conducted by solving the incompressible Reynolds Averaged Navier-Stokes equations, shows that both parameters can significantly affect flow characteristics and its radiated sound to the far field, which is calculated through the Ffowcs-Williams and Hawkings acoustic analogy. Three-dimensional compressible Delayed Detached-Eddy simulations, on similar cases are performed. It is shown that as the leg-door angle increases, the wake width increases and the noise spectra peak tends towards lower frequencies and at higher levels. It is also shown that the leg-door element becomes a more significant noise source, as the interaction at high leg-door angle can cause the turbulent intensity in the wake from the cylinder to decreases. All numerical works were performed at a Mach number of 0.2 and a Reynolds number based on the cylinder diameter of $1.7 \times 10^{6}$. The experimental data was conducted at a Mach number of 0.09 and Reynolds number of $0.202 \times 10^{6}$ and consists of time averaged velocity field statistics and an on surface microphone spectra. The experiment was conducted for three leg-door angles. The velocity and turbulent intensity along the wake is compared to the 3-D simulations to show agreements.
\end{abstract}

\section{Nomenclature}

Roman

$D \quad$ Cylinder diameter, $\mathrm{m}$

$G \quad$ Gap width ratio

$I_{i i} \quad$ Turbulent intensity, $\sqrt{\overline{u_{i} / u_{i}}} / U_{\infty}$

$M \quad$ Mach number

Re Reynolds number

St Strouhal number, $f D / U_{\infty}$

$T$ Temperature, $\mathrm{K}$

$|u| \quad$ Velocity magnitude, $\mathrm{m} / \mathrm{s}$

$U_{\infty} \quad$ Free stream speed, $\mathrm{m} / \mathrm{s}$

$\left|x_{\text {obs }}\right|$ Observer position from source, $\mathrm{m}$

\section{Greek}

$\alpha_{D} \quad$ Door angle of attack, degrees

$\theta_{\text {obs }} \quad$ Observer angle, degrees

*PhD student, Aeronautics, Astronautics and Computational Engineering, rjf106@soton.ac.uk

${ }^{\dagger}$ Lecturer, Aeronautics, Astronautics and Computational Engineering, Member AIAA.

${ }^{\ddagger}$ Lecturer, Aeronautics, Astronautics and Computational Engineering, Member AIAA. 


\section{Introduction}

$T^{\text {HE }}$ leg-door and main strut of an aircraft main landing gear (MLG) are in close proximity. This type of problem can be modeled by the interaction between a cylinder placed near a flat plate. Studies have shown that under close proximity to a solid boundary the wake intensity behind the cylinder will decrease. ${ }^{1-3}$ The change in the shedding intensity was found to correlate to a non-dimensional gap width $G$ for various types of geometries. The parameter $G$ is defined as the ratio of the gap dimension (between the cylinder and the plane boundary) to the cylinder diameter. The general aeroacoustic impact of this effect is an expected reduction in the far field sound pressure level (SPL), due to the reduced turbulence level in the wake.

Nishino and Roberts ${ }^{4}$ investigated the near wake stability characteristics of a quasi-laminar flow behind a 2-D bluff body placed near and parallel to a plane boundary. They found that the ratio of the centerline wake velocity to the cylinder edge velocity to be an important parameter determining the cestation of the primary vortex shedding mode.

Hutcheson and Brooks ${ }^{5}$ conducted an experimental investigation on the effects of the gap width ratio $G$, on the far field acoustics. Their model consisted of a cylinder and a flat plate, more representative of a MLG and was tested under two gap configurations; $G=0$ and $G=0.133$. The far field noise spectra showed that the changes between a flush-mounted and finite-gap configuration could cause a difference in the peak SPL by $20 \mathrm{~dB}$ when there is a uniform inflow. With a grid-induced turbulent inflow, the difference in the peak SPL is around $10 \mathrm{~dB}$. The estimated Strouhal number range, across which the changes in the spectra occur, is $0.065<S t<0.30$. This estimation is based on the cylinder diameter, and the cited Mach number and Reynolds number.

Numerical work on noise generation mechanisms around a MLG system has been focusing on simplified geometries to reduce grid complexities. ${ }^{6,7}$ Some studies have included the MLG leg-door as part of the model. $^{8-10}$ However, the changes to the unsteady flow field due to the presence of the leg-door was not studied.

An experimental assessment on the effect of the MLG leg-door has also been studied on a high fidelity MLG model where two sets of configurations, with and without the leg-door, were studied. ${ }^{11}$ The effect of the leg-door was shown to yield some changes to the noise directivity patterns but the experiment did not test the effects on varying the door angle or the gap configuration.

The MLG of some long range civil aircrafts have large and angled leg-doors where the ratio of the leg-door height to the main strut height can be approximately 0.7 . This suggests that any significant changes to the unsteady flow field from the leg-door interaction could affect a significant portion of the MLG. Additionally, the effect of an angled leg-door may also be considered for an aircraft landing in cross wind.

Noise prediction models are useful to the aircraft manufacturer to assess model designs at reduced costs. The development of aircraft landing gear noise prediction models has come to include greater details about the MLG. ${ }^{12}$ The current treatment of the leg-door as a noise source is typically set as a trailing edge source, which has recently been considered as an insignificant source affecting the overall noise from a MLG. ${ }^{13}$

The current work aims to determine the impact of an angled leg-door on the far field noise due to the aerodynamic interaction on a simplified model. In Section II a parametric 2-D and unsteady Reynolds Averaged Navier-Stokes (RANS) study is outlined using a simplified door and main strut configuration. In Section III selected cases studied further in 3-D using a Delayed Detached-Eddy Simulation (DDES) methodology, for an infinite span geometry, are presented. In Section IV the experimental tests are presented to validate the numerical work.

\section{Two-Dimensional Parametric Study}

A simplified door and cylinder interaction geometry was defined for a parametric study and is shown in Figure 1. The door angle $\alpha_{D}$, and the gap between the door and the cylinder element were selected as the variables for this study. The door angle was varied between $0<\alpha_{D}<10.7$ degrees. The gap between the two elements was set by a non-dimensional parameter $G$, defined as the ratio of the minimum gap distance to the cylinder diameter, and ranged from $0.087<G<0.2$.

A second order finite volume code was used to solve the unsteady incompressible Reynolds Averaged Navier-Stokes (RANS) equations with the $k-\omega$ SST turbulence model, and the Ffowcs-Williams and Hawkings $(\mathrm{FW}-\mathrm{H})$ equation was used to calculate the far field acoustic signals. The turbulence model was selected by reviewing some previous works on 2-D flows around bluff bodies. ${ }^{14,15}$ The geometry is representative of a 


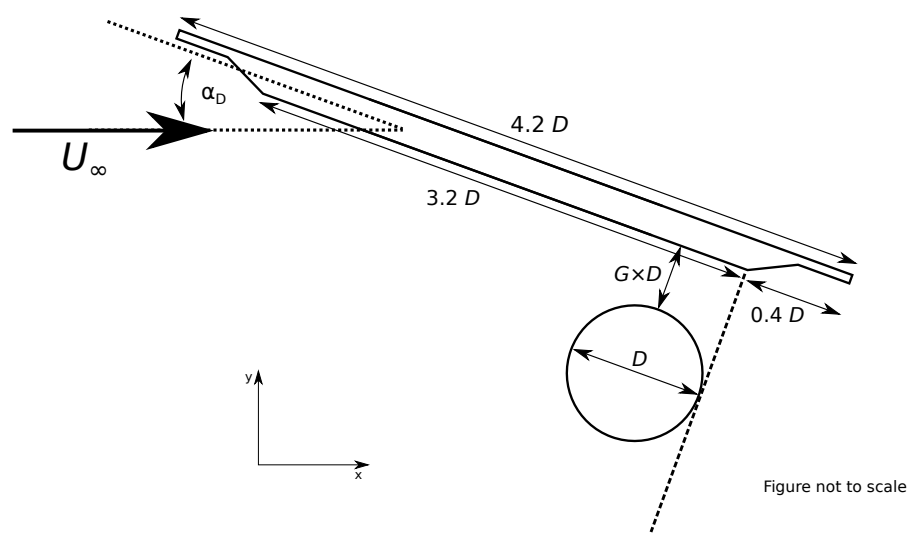

Figure 1. Geometric 2-D outline for the study.

full scale MLG model with a Reynolds number based on the cylinder diameter of $R e_{D}=1.7 \times 10^{6}$. The free stream velocity was set to a Mach number of $M_{\infty}=0.2$ at a temperature of $T_{\infty}=288.16 \mathrm{~K}$.

The use of an incompressible solver for aeroacoustic application is valid for compact sources where the acoustic wave length is much larger than the geometry. In these cases the interaction between the acoustic wave and the geometry is small so the far field acoustic signals are highly correlated to the unsteady flow field. Furthermore, the RANS methodology resolves only the dominant, low frequency scales and so this methodology is thought to be sufficient for a low-cost preliminary study.

The FW-H method ${ }^{16}$ was used to predict the far field sound $\left(\left|x_{o b s}\right| / D \approx 300\right)$ using the pressure and velocity fluctuations recorded on the surface of door, the cylinder, and an off body surface which encloses the entire model and a part of the wake. The observer positions are referred to by their observer angle $\theta_{\text {obs }}$ which is measured anti-clockwise from the downstream direction. The $\theta_{o b s}=0^{\circ}$ and $\theta_{o b s}=90^{\circ}$ cases indicate an observer positioned directly downstream, and overhead on the door side of the model, respectively.

A grid convergence study was conducted on one of the test cases to determine the grid parameters for the wake region. The boundary layer region was refined individually for each case to ensure that the wall resolution was adequate and controlled by the wall $y^{+}$which averaged around $y^{+} \approx 2.4$ with a maximum of $y^{+}=4.5$.

\section{II.A. Results}

Two baseline configurations, an isolated cylinder and a door element, were first tested with RANS. Results concluded that the flow behind an isolated door element at zero degrees angle of attack is significantly weaker than from an isolated cylinder. The primary noise generation mechanisms for these two cases is the vortex shedding.

A further set of cases were run for $0<\alpha_{D}<10.7$ and $0.087<G<0.2$ to study the interaction between the two elements. Figure 2 shows the radiated far field directivity patterns for the additional cases. The general trends for the two configurations at $G=0.15$ and $G=0.2$ show that as the door angle increases, the sound level decreases. This behavior is thought to be caused by the suppression of the cylinder shedding which is the primary noise source. In the case of $G=0.087$ there is a different trend where increasing the door angle first decreases the noise level, however further increases over $\alpha_{D}>5$. This secondary trend is not present when the gap is larger and it may indicate a condition where the door element becomes a significant noise source as it forms large structures which are paired with the cylinder structures to form a more coherent shedding pattern.

The suppression of the vortex shedding behind a cylinder due to the interaction with a flat plane boundary has been studied extensively. Previous studies show that the shedding intensity behind the cylinder decreases with smaller gap width ratios $G$. The setup of the presented cases, illustrated in Figure 1, shows that increasing the door angle at a fixed gap width changes the projected gap height to the free stream flow. Increasing the door angle effectively decreases the gap width ratio seen by the free stream flow. The decrease in the projected gap height with greater door angle causes a greater acceleration of the fluid through the gap resulting in the momentum of the flow around the two sides of the cylinder to differ more. The difference in the flow velocities around both sides of the cylinder has been noted as a significant parameter which 


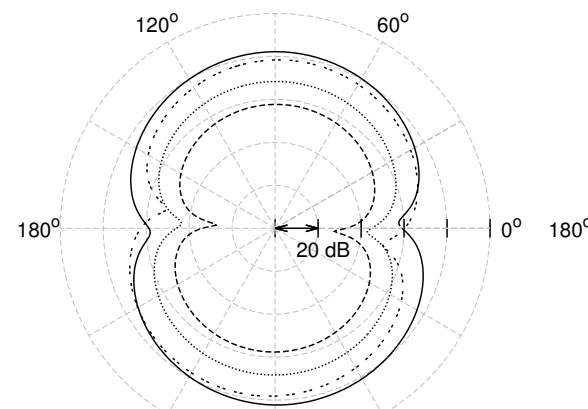

$240^{\circ}$

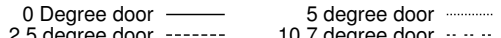

(a) $G=0.087$ Door FW-H

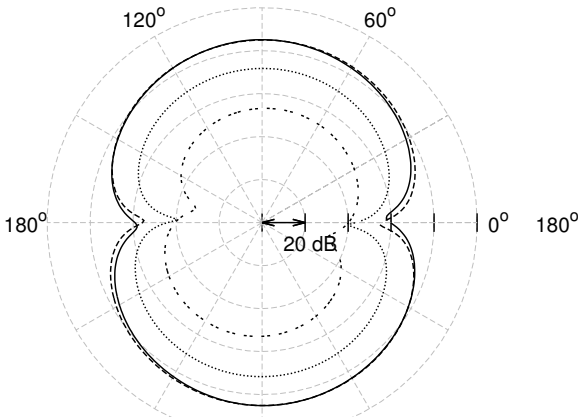

$240^{\circ}$

$300^{\circ}$

0 Degree door — $\quad 5$ degree door ............. 2.5 degree door

(d) $G=0.15$ Door FW-H

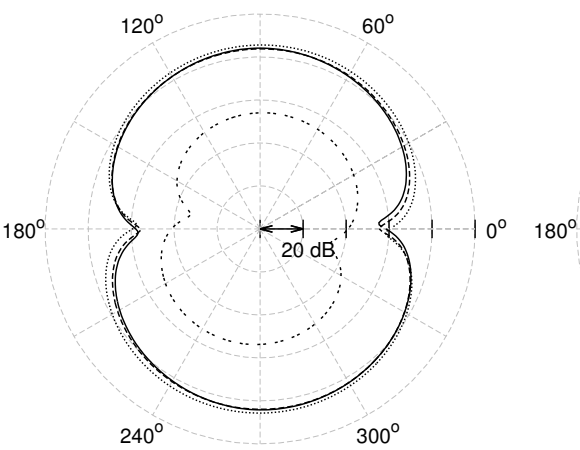

0 Degree door
2.5 degree door 5 degree door $\cdots \cdots \cdots \cdots . .$.
10.7 degree door $\ldots . .$.

(g) $G=0.2$ Door FW-H

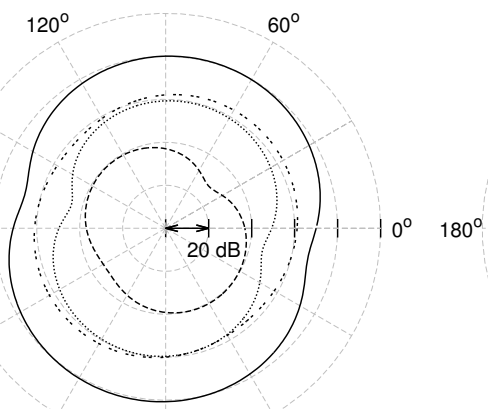

$240^{\circ}$

$300^{\circ}$

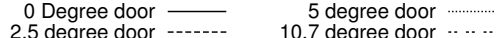

(b) $G=0.087$ Cylinder FW-H

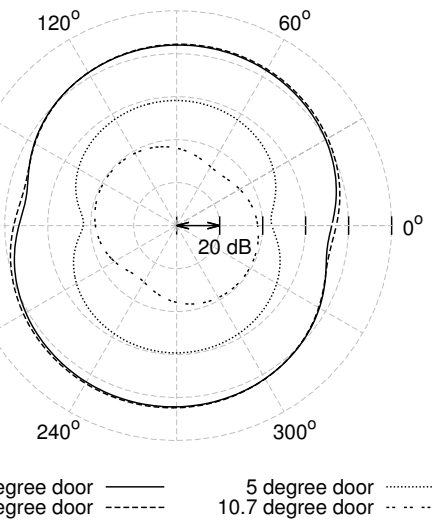

(e) $G=0.15$ Cylinder FW-H

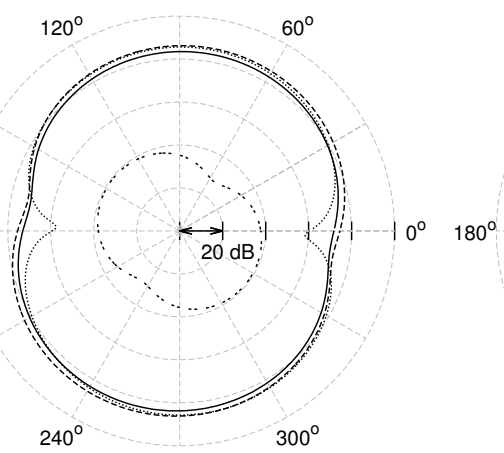

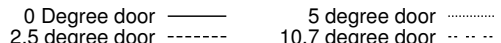

(h) $G=0.2$ Cylinder FW-H

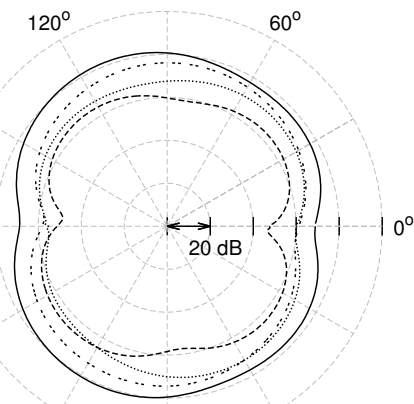

$240^{\circ}$

$300^{\circ}$

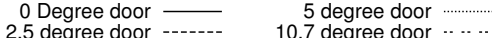

(c) $G=0.087$ Off-body FW-H

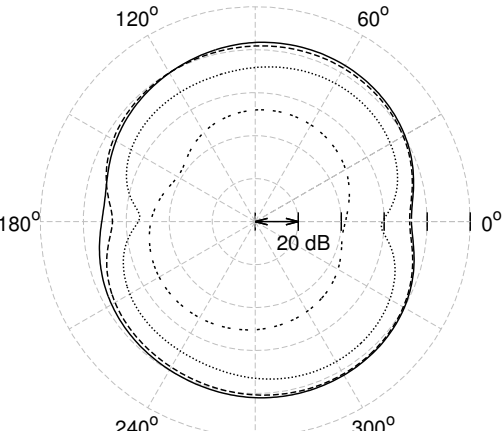

$240^{\circ}$

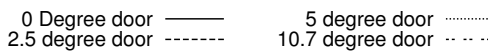

(f) $G=0.15$ Off-body FW-H

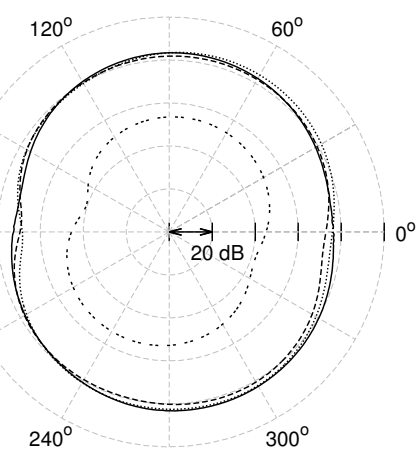

0 Degree door - 5 degree door 2.5 degree door ------- $\quad 10.7$ degree door ......

(i) $G=0.2$ Off-body FW-H

Figure 2. The far field OASPL directivity patterns from the 2-D simulations. 
determines the vortex shedding suppression. ${ }^{4}$ The differences in the fluid momentum may make flow around one side of the cylinder less prone to disturbances, reducing the strength of the vortex shedding pairing, and reducing the shedding intensity.

The 2D-parametric study indicates that the door influences the far field acoustics strongly. The primary observation is that the wake intensity behind the cylinder element changes with the door configuration.

\section{Three-Dimensional Simulations}

Results from the 2-D study showed similar trends in the changes of the OASPL for varying door angle at constant gap width ratios at $G>0.087$. The trend for the closest interaction case shows that as the door angle is increased, the overall sound level could increase. For this reason the case for $G=0.087$ was chosen for further investigation.

The test cases for the 3-D study are outlined in Table 1, where the geometry is a spanwise extrusion of the 2-D cases by a fixed length of $3 D$ and with a spanwise grid resolution, measured as the ratio of the spanwise grid size to the cylinder diameter, of $\Delta z / D=0.05$. The 3-D meshes were generated from the best 2-D meshes after a grid independent study and the scale of the model and the free stream conditions were kept the same as in the 2-D studies.

\begin{tabular}{ccll}
\hline \hline Case name & Case description & Gap width ratio, $G$ & $\begin{array}{l}\text { Door angle of attack, } \alpha_{D} \\
\text { (degrees) }\end{array}$ \\
\hline Bcase 1 & Baseline, isolated cylinder case & N/A & N/A \\
Bcase 2 & Baseline, isolated door case & N/A & 10.7 \\
Icase 1 & Intercation case 1 & 0.087 & 0 \\
Icase 2 & Intercation case 2 & 0.087 & 5 \\
Icase 3 & Intercation case 3 & 0.087 & 10.7 \\
\hline
\end{tabular}

Table 1. Outline of the 3-D cases run using the high order code.

\section{III.A. Numerical method}

An in-house high order finite difference code was used to solve the compressible Navier-Stokes equations using the DDES method. ${ }^{17}$ An implicit, prefacted and optimized fourth order accurate scheme is used for the interior points and is closed by third order accurate, explicit schemes. ${ }^{18}$ The time integration scheme follows a second order, fully implicit, dual-time stepping approach with a Newton-like sub-iteration ${ }^{19}$ and the numerical stability is provided by an explicit sixth order filter. The overall accuracy of the code has been demonstrated for benchmark cases to fourth order. ${ }^{20}$ A buffer zone condition ${ }^{21}$ is applied on the domain boundaries where numerical reflections are undesirable.

A non-dimensional time step, based on a unit reference length and the free stream sound speed, of $\Delta t^{*}=3 \times 10^{-4}$ was used. The maximum Courant number (based on the local cell dimension and the local sound convection speed) is 20 which is located within the boundary layer mesh. The Courant number outside the boundary layer is low enough to consider the majority of discretization error to be from the spatial schemes.

The test cases form a fully developed flow field by the time the free stream flow has convected by 20 cylinder diameters, and afterwards the FW-H data, sampled every 100 time steps, was collected over a similar time period.

Figure 3 shows the mesh in the near field region and the multi-block boundaries. The instantaneous flow features colored by velocity magnitude are also shown in Figure 3. The grid in the near field region was set to a spacing aimed to resolve acoustic waves up to $4 \mathrm{kHz}$. This calculation was based on the maximum resolution characteristics for the spatial scheme used for the interior points. ${ }^{18}$

\section{III.B. Results}

The changes to the unsteady flow behind the model with varying door angle is shown by Figure 4, where the flow unsteadiness is outlined by the mean turbulent kinetic energy (TKE) field and the streamlines based 


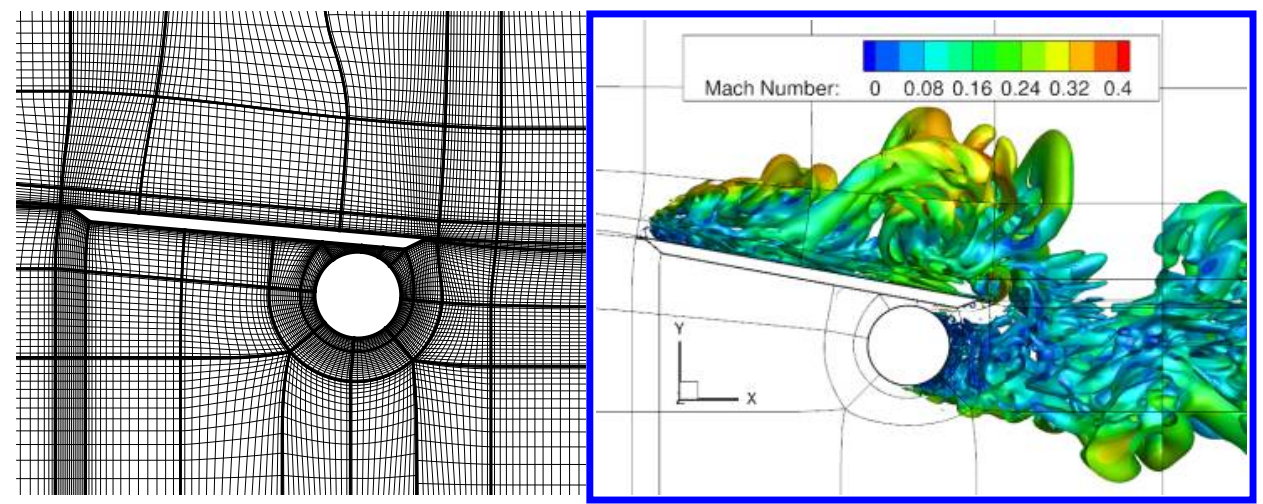

Figure 3. The grid for Icase2 (left) showing every third grid line and outlining the block boundaries. The instantaneous surface plot for constant Q-criterion colored by the local Mach number for Icase3 (right).

on the mean velocity field. The mean TKE field is computed using the resolved Reynolds stresses along the principal axis, and is made non-dimensional using the free stream velocity. Figure 4 shows that the leading edge bevel on the lower surface of the door (at $x / D \approx-3)$ may form a region of adverse pressure gradient. At the lowest door angle, this generates a separation bubble that spans from $-3<x / D<0.5$. Turbulent structures are produced within this region and impinge on the cylinder surface which could generate interaction noise. At higher door angles the inflow impinges more on the lower surface of the door and a more favorable pressure gradient reduces the size of the separation bubble. A secondary separation bubble is also introduced around $-1.2<x / D<0.8$ where the influence from the cylinder is greater. This separation bubble is formed due to the adverse pressure gradient near the stagnation region on the cylinder face.

The recirculation region on the upper surface of the door is also shown in Figure 4. The size of the separated flow along the upper surface of the the door is highlighted by the streamline patterns, which grows with increasing door angle. This pattern is expected as the model becomes less streamlined. A peak in the mean TKE occurs around the trailing edge of the door, at a high angle of attack, where a strong shear layer is present. This shear layer is formed by the interaction of two features; the separated flow along the upper surface of the door, and the accelerated flow through the gap which is deflected upwards due to the low pressure generated by the angled door.

The nine components of the production term in the mean TKE transport equation was studied to understand the mechanisms which causes the TKE peak towards the door trailing edge. It was found that the dominant contributions comes from the terms with a $y$-derivative. It should however be noted that the other terms are more significant in other regions of the flow field. Therefore, a reduction in the peak intensity in this region could be achieved by reducing the door angle, or by increasing the gap distance.

The parametric 2-D study, presented in Section II, found that the point at which the noise level would diminish correlates better to a separate length parameter termed as the project gap height. This is confirmed by Figure 4, as the door angle increases the stagnation point moves along the cylinder towards the door surface. This trend has been observed by Bearman and Zdrakovich ${ }^{2}$ on an experiment studying the flow around a cylinder near a flat plane boundary. As greater similarities are noted by using the projected gap height, it is thought that this parameter may be more significant in affecting the wake pattern.

Figure 4 also shows the decrease in the wake intensity immediately behind the cylinder element as the door angle is increased. This will be further discussed in Section IV.

The far field acoustic signals were calculated with the same method as used in the 2-D work. The integration surfaces were placed within the near field region that consists of a fine mesh and follows the grid lines. The integration surfaces for the Icase 2 is shown in Figure 5 and the other cases have similar surface placements. The FW-H method requires that the body encloses all acoustic sources, from which a free field Green's function can be appropriately applied. Figure 5 shows the close placement of the off-body surface to the lower surface of the door, which may cause issues with the validity of using a free field Green's function. There may be sound scattered around the cylinder which reflects off the door surface, thereby suggesting that the surface may not be enclosing all sources.

The directivity patterns of OASPL for the baseline, cylinder (BCase1) and angled door (BCase2) cases 

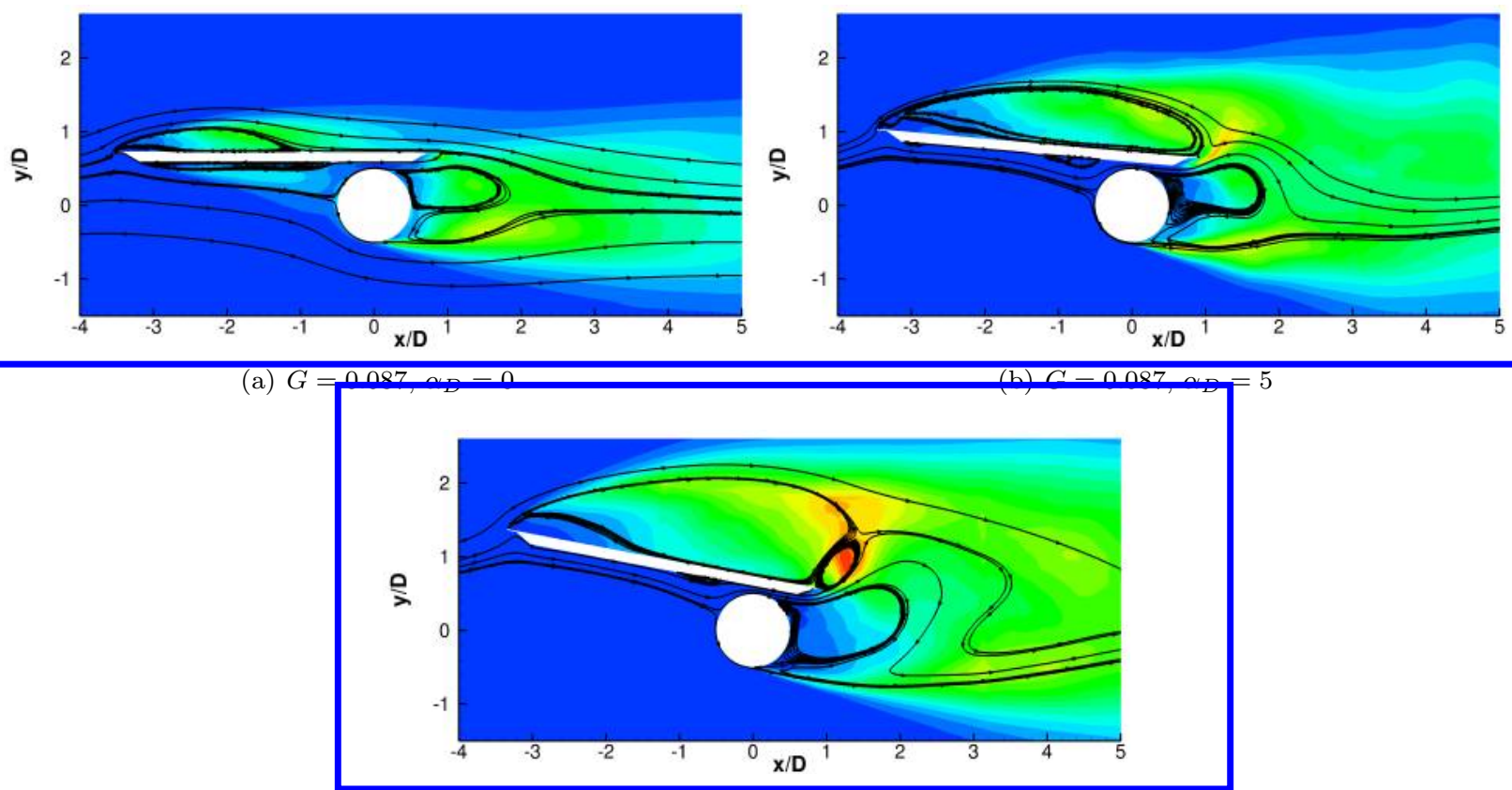

(c) $G=0.087, \alpha_{D}=10.7$

Figure 4. Contour plot for the non-dimensional mean turbulent kinetic energy field for the three interaction cases. The scalar field is ranged from 0 to 0.25 in 18 levels and highlighted with streamlines.

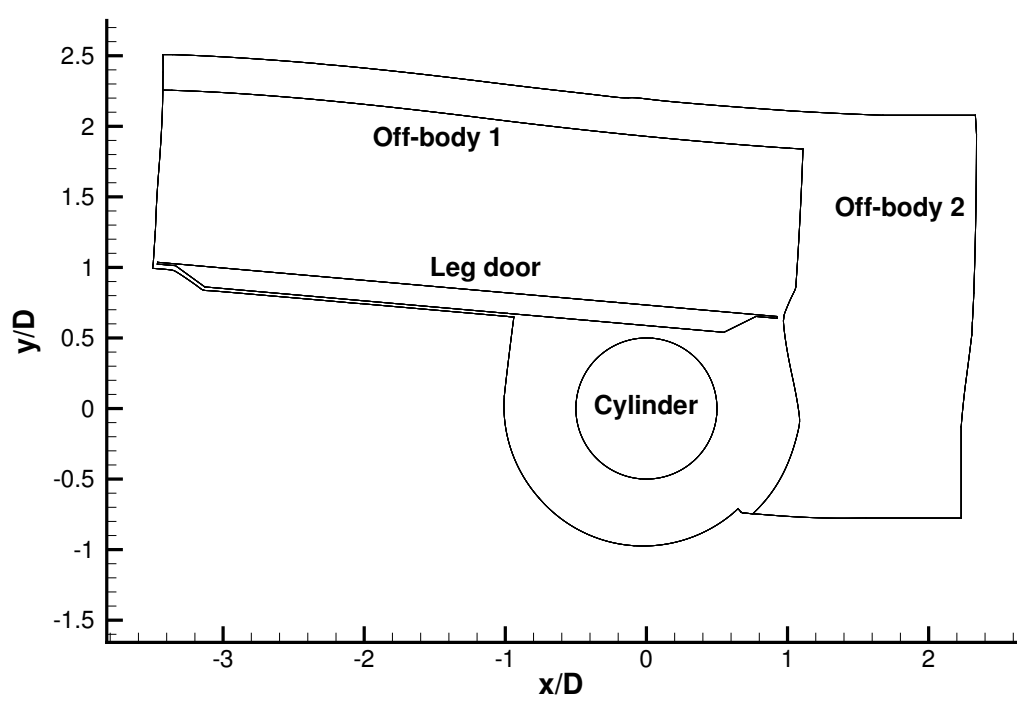

Figure 5. FW-H integration surface placement for Icase2. Four surfaces exist; on the door, cylinder and two off body surface enclosing the entire model and part of the wake region. 
yields a dipole behavior. The noise spectra for a far field observer at $\theta_{o b s}=90^{\circ}$ for both cases is shown in Figure 6. All spectral plots are shown as a function of the Strouhal number, defined using the cylinder diameter and the free stream velocity. The spectra show a peak which corresponds to the primary vortex shedding mode to each of the baseline cases. The noise generated from the angled door is greater than from an isolated cylinder. The isolated angled door is at $\alpha_{D}=10.7$ which causes a fully detached flow over the upper surface and the wake region forms across the entire upper surface and close to the body. This feature is thought to be the main reason why the isolated door case is louder than the isolated cylinder case.

The noise spectra for an observer in the same position $\left(\theta_{o b s}=90\right)$, for all three interaction cases, using the most outer-edge off-body integration surface is shown by Figure 7.

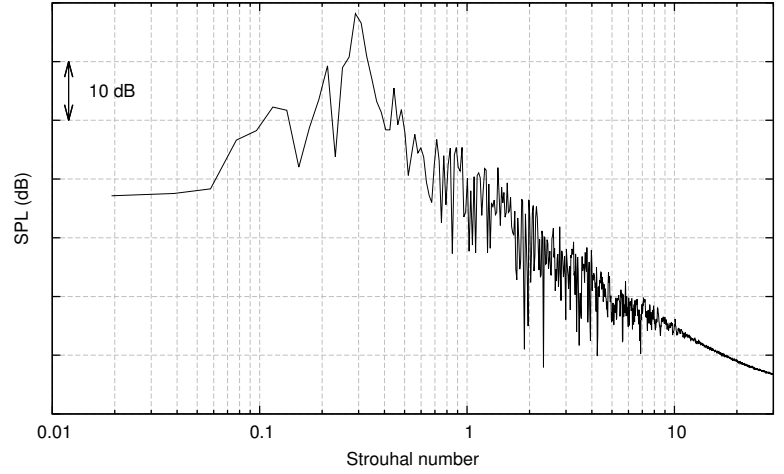

(a) Isolated cylinder

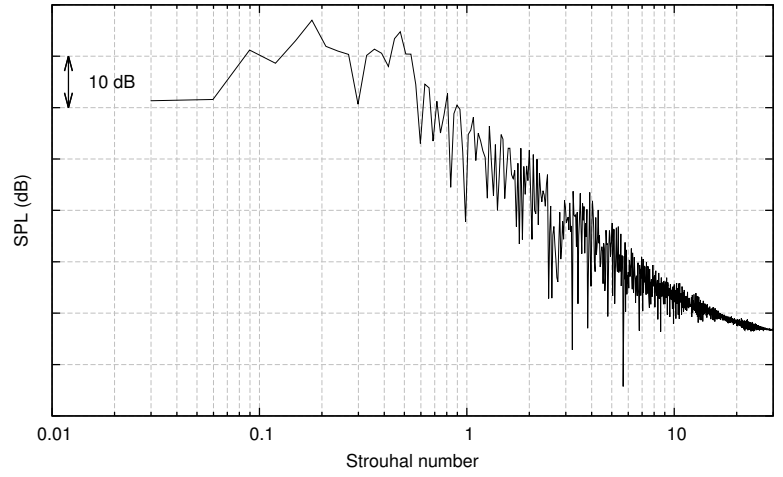

(b) Isolated door

Figure 6. Noise spectra for the far field acoustic signal for an observer at $\theta_{o b s}=90^{\circ}$.

The directivity pattern for the interaction case at $\alpha_{D}=0$ shows some asymmetry due to the shielding effect of the door. A shadow region forms, which can be up to $3.5 \mathrm{~dB}$ quieter. This asymmetry diminishes as the door angle is increased due to the significantly large noise generated by the detached flow from the angled door.

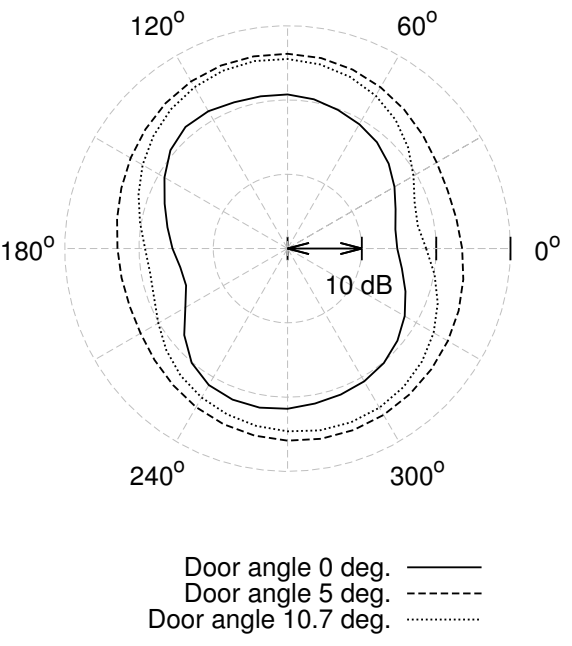

(a) OASPL directivity pattern

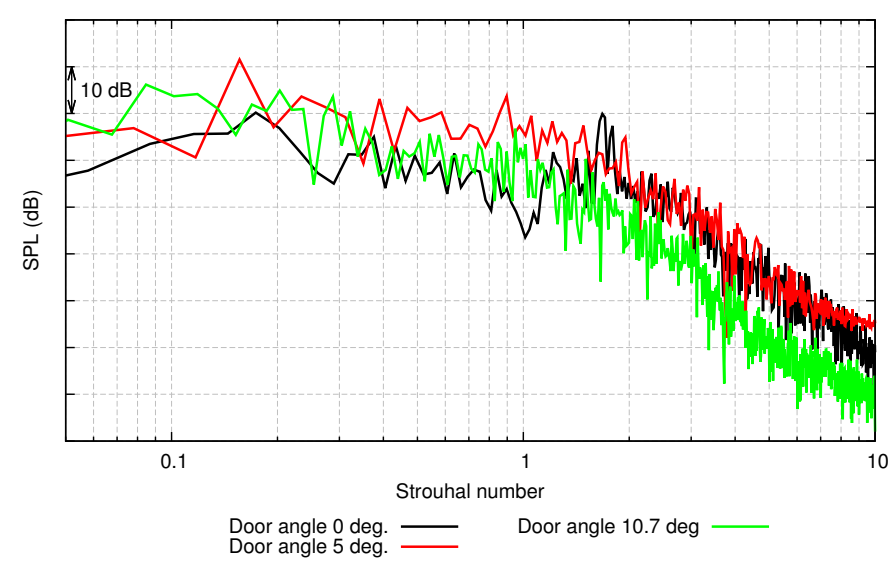

(b) Noise spectra for $\theta_{o b s}=90^{\circ}$

Figure 7. Aeroacoustic result calculated using the largest off-body FW-H surface.

The noise spectra shown on the right in Figure 7 show the trend in the dominant tone tending to lower frequency with increasing door angle. These tones occur at frequencies lower than the vortex shedding from the isolated cases. This trend is expected due to the increased width of the wake profile indicated in Figure 4. The trend could be correlated better to the projected height of the entire interaction model, as this is the only geometric length scale that increases with door angle.

Figure 8 shows the noise spectrum for a far field observer at a position of $\theta_{o b s}=90$ and calculated using 


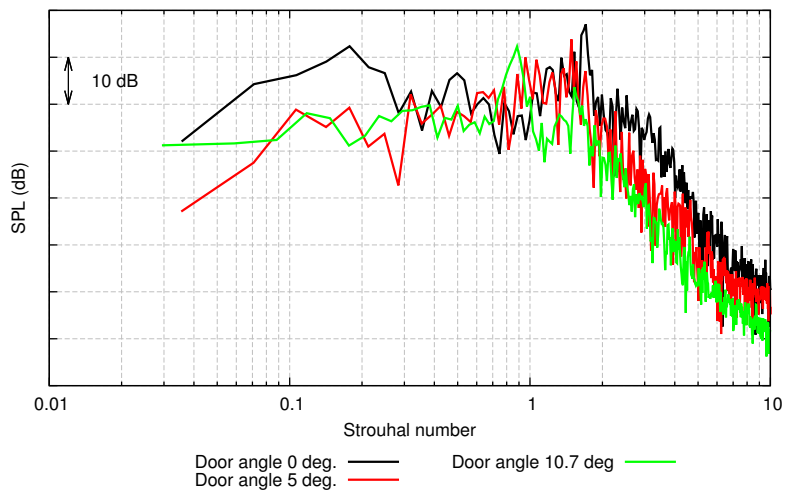

(a) Cylinder on-body FW-H

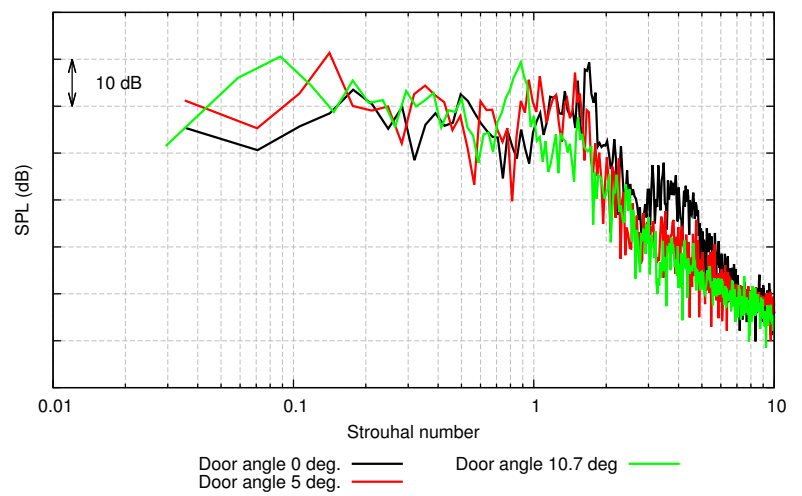

(b) Door on-body FW-H

Figure 8. Far field noise spectra for an observer at $\theta_{o b s}=90$, calculated using the on-body surface data only.

either the on-body door or cylinder surface data. These calculated results do not resemble the physical far field noise, as the free field Green's function used does not account for effects such as the shielding effect of the door on the radiated sound from the cylinder. Instead, these results can be used to qualitatively assess the changes to the sources in various interaction settings. As this data is collected along no-slip boundaries, only pressure and density fluctuations are considered. Figure 8 shows that as the door angle increases, the fluctuations on the cylinder tends to peak at lower frequencies and becomes weaker. However, the fluctuation intensity along the door surface increases, making it a more significant noise source, and tends to peak at lower frequencies as the door angle increases. The vortex shedding from the cylinder element $(S t \approx 0.2)$ seems to weaken under the interaction, however the overall noise level increases, due to the significance of the door element as a noise generator. The tendency to lower frequency is associated to the widening of the wake at greater door angles.

Differences in the behavior of OASPL with increasing door angle are present between the 2-D and 3-D simulations. The primary difference is the increased impact of the door element as a noise source at greater door angles which is only predicted better by the 3-D simulations. The change in OASPL between $\alpha_{D}=0$ and $\alpha_{D}=10.7$ is around $20 \mathrm{~dB}$ from the RANS simulations and $10 \mathrm{~dB}$ from the 3 -D results. This difference is due to the reduced coherency of the flow perturbations along the FW-H surface for the 3D DDES simulations.

\section{Experimental Work}

Experiments were conducted at the University of Southampton $2.1 \times 1.5$ meter low speed wind tunnel to validate some of the 3-D simulation findings. An experimental model based on the same geometry, as shown in Figure 1, was constructed with a spanwise length of $10 \mathrm{D}$ and fitted with an on-surface microphone in the mid-span of the cylinder model at $\theta=90^{\circ}$. The door model leading and trailing edges were simplified and the difference can be seen within Figure 12. The major flow physics are thought to be negligibly influenced by the door edge modification.

The model surface was gritted to simulate higher Reynolds number to provide flow features more comparable to the numerical results. Figure 9 outlines the model as it was set in the test section with the laser outlining the span-wise position of the target plane.

The velocity fields were obtained using particle image velocimetry (PIV). Data was collected at a free stream velocity of $U_{\infty}=30$ onto on a $300 \times 300 \mathrm{~mm}$ window with a $2048 \times 2048$ pixel a camera. Pairs of images taken with a specified time spacing are cross correlated to determine the particle displacement which outlines the velocity field. A database of 500 images were collected at a rate of $3.63 \mathrm{~Hz}$ and the final processed velocity field had a model resolution of $0.046 D \times 0.046 D$, similar to another PIV study. ${ }^{22}$ Table 2 outlines the test cases and the timing setup. 


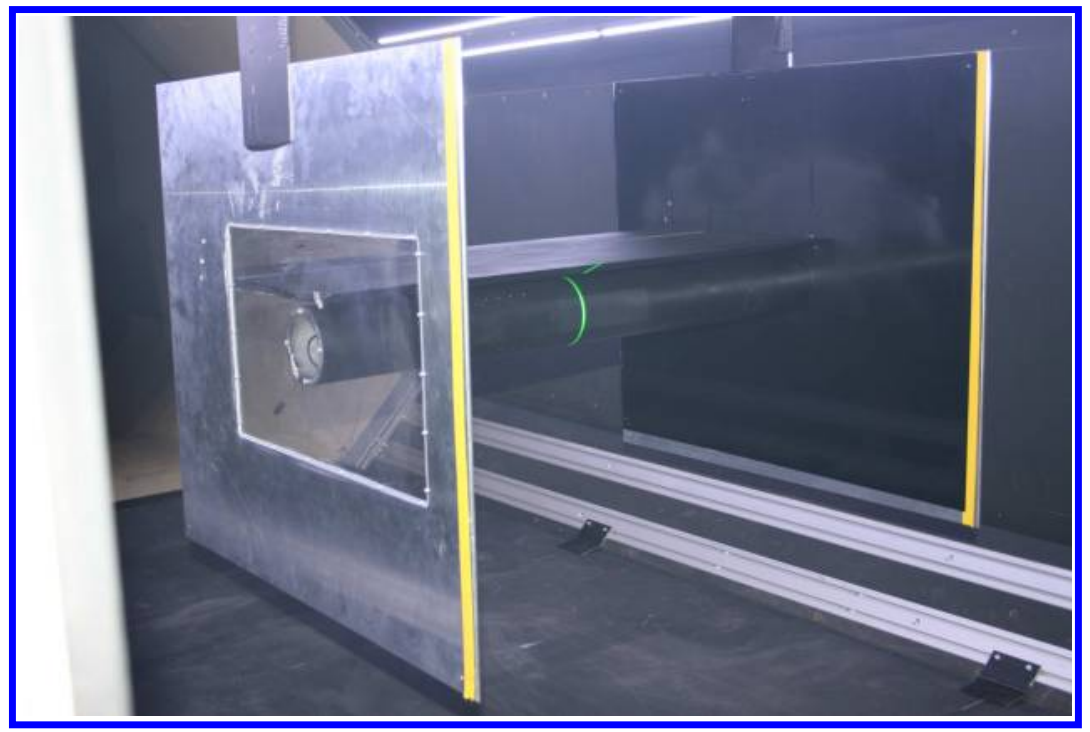

Figure 9. Experimental model setup in the test section, at the University of Southampton $7 \times 5$ foot wind tunnel. Green laser indicates the span-wise position of the PIV target plane.

\begin{tabular}{ccc}
\hline \hline Gap width ratio & Door angle (degrees) & PIV capture time, $\Delta t(\mu s)$ \\
\hline 0.087 & 0 & 37.5 \\
0.087 & 5 & 38 \\
0.087 & 10.7 & 45
\end{tabular}

Table 2. PIV camera timing setup for all tested configurations in the experiment at $U_{\infty}=30$.

\section{IV.A. Experimental results}

The 2-D velocity and turbulent intensity profiles, across the wake $(-1<y / D<1)$ at two downstream positions $(x / D=\{1.5,2.0\})$, are compared to determine the effect of varying the door angle. The trends for the velocity magnitude are shown in Figure 10. The unsteady features in the wake region are characterized by the high turbulent intensities, calculated using two of the principal Reynolds stresses, and the trends are shown in Figure 11.

Figure 10 shows that as the door angle increases, the wake width behind the cylinder element increases. The velocity profile along the wake of the entire model was not captured within the available window for the PIV. The velocity magnitude profiles also show a secondary profile for $x / D=1.5$ within the region of $0.5<y / D<0.8$ which highlights the accelerated flow through the gap between the leg-door and the cylinder elements. It should be noted that the accelerated flow may be deflected upwards into the low pressure region formed by the angled door.

Although the wake is noted to become wider, the turbulent intensity immediately behind the cylinder decreases with increasing door angle. This may suggest that the primary vortex shedding behind the cylinder element is being suppressed. The data in Figure 11 also suggests that the turbulent intensity behind the door trailing edge, at $y / D>0.75$, is increasing with greater door angle.

\section{IV.B. Comparison to numerical simulation}

In this section the experimental data is compared to the results obtained from the 3-D simulations. All variables that are compared between the experiments and simulation are done on a non-dimensional axis based on the free stream velocity, to account for the difference in the model scale. The time-averaged velocity magnitude fields, for two of the three interaction cases, are compared between experiment and simulation in Figure 12.

The far field acoustic signals from the 3-D simulations were calculated using the near field unsteady flow data, which can be characterized by the turbulent intensities, which are compared along the wake $(-1<y / D<1)$ at two downstream locations $x / D=\{1.5,2.0\}$ and shown in Figures 13 and 14. The overall 


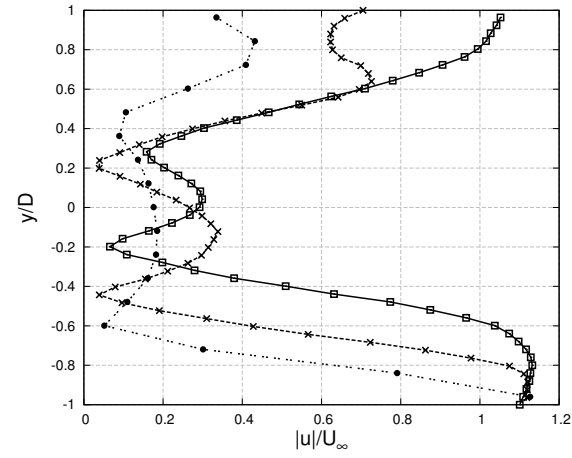

Door at 0 deg $\rightarrow$ Q-
Door at 5 deg $---x---\quad$ Door at $10.7 \mathrm{deg} \cdots .$.

(a) $x / D=1.5$

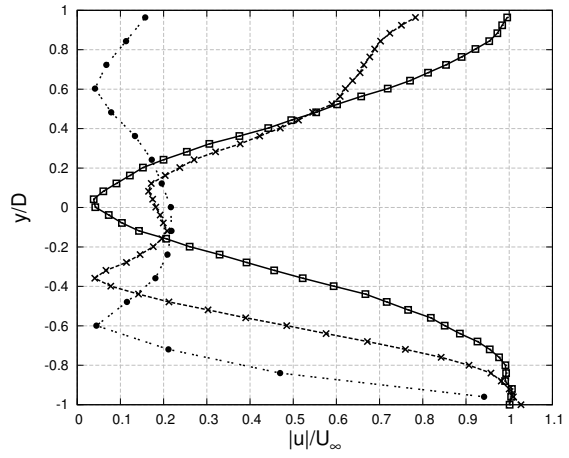

Door at 0 deg $\rightarrow \square$
Door at 5 deg $-\cdots---\cdots$$\quad$ Door at $10.7 \mathrm{deg} \cdots .$.

(b) $x / D=2$

Figure 10. The time-average velocity magnitude wake profile at the specified downstream locations.

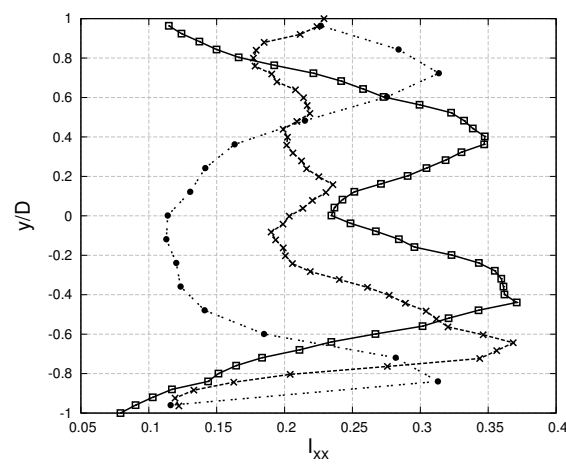

Door at 0 deg $\rightarrow-\square$
Door at 5 deg $---x---$

(a) $x / D=1.5$

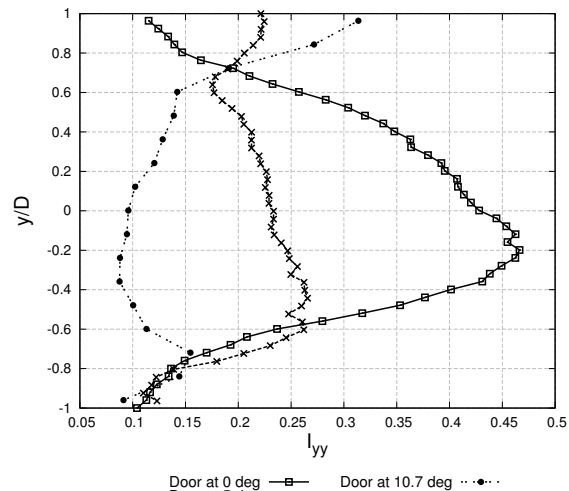

Door at 0 deg $\rightarrow-\square$
Door at 5 deg $----x--$

(c) $x / D=1.5$

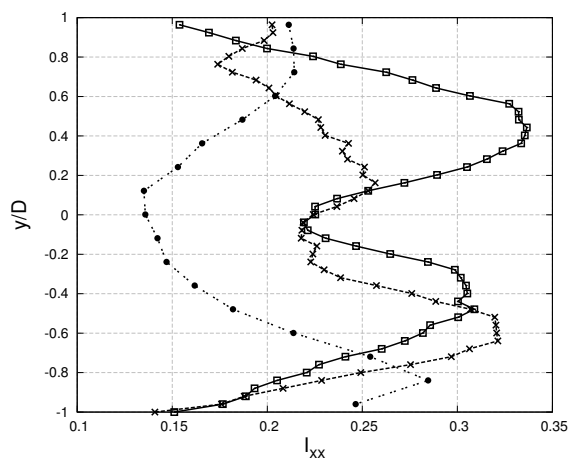

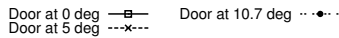

(b) $x / D=2$

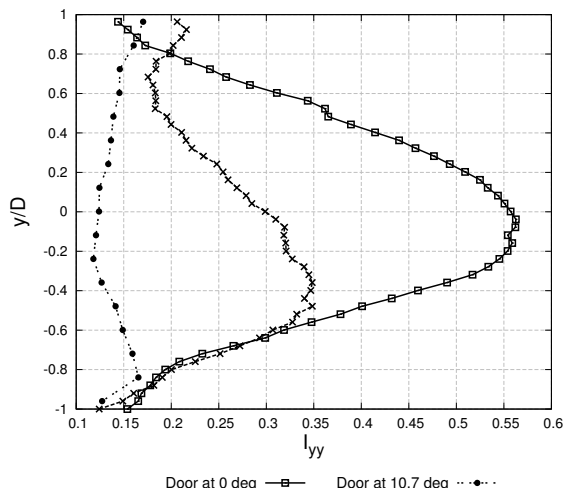

Door at 0 deg $\rightarrow \quad$ Door at $10.7 \mathrm{deg} \ldots . .$.
Door at 5 deg --- -.-.

(d) $x / D=2$

Figure 11. The turbulent intensity wake profile based on $\overline{u / u \prime}$ (top) and $\overline{v / v \prime}$ (bottom) at the specified downstream locations.

\section{1 of 17}




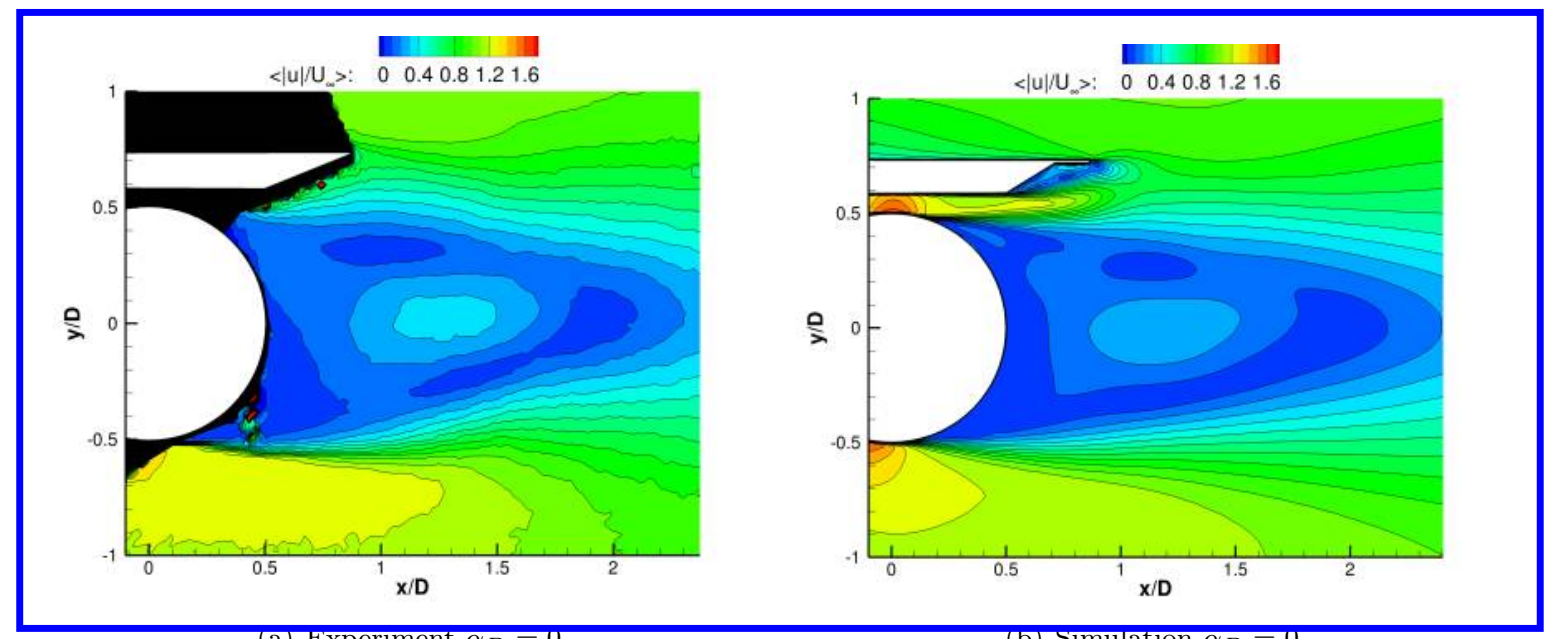

(a) Experiment $\alpha_{D}=0$

(b) Simulation $\alpha_{D}=0$

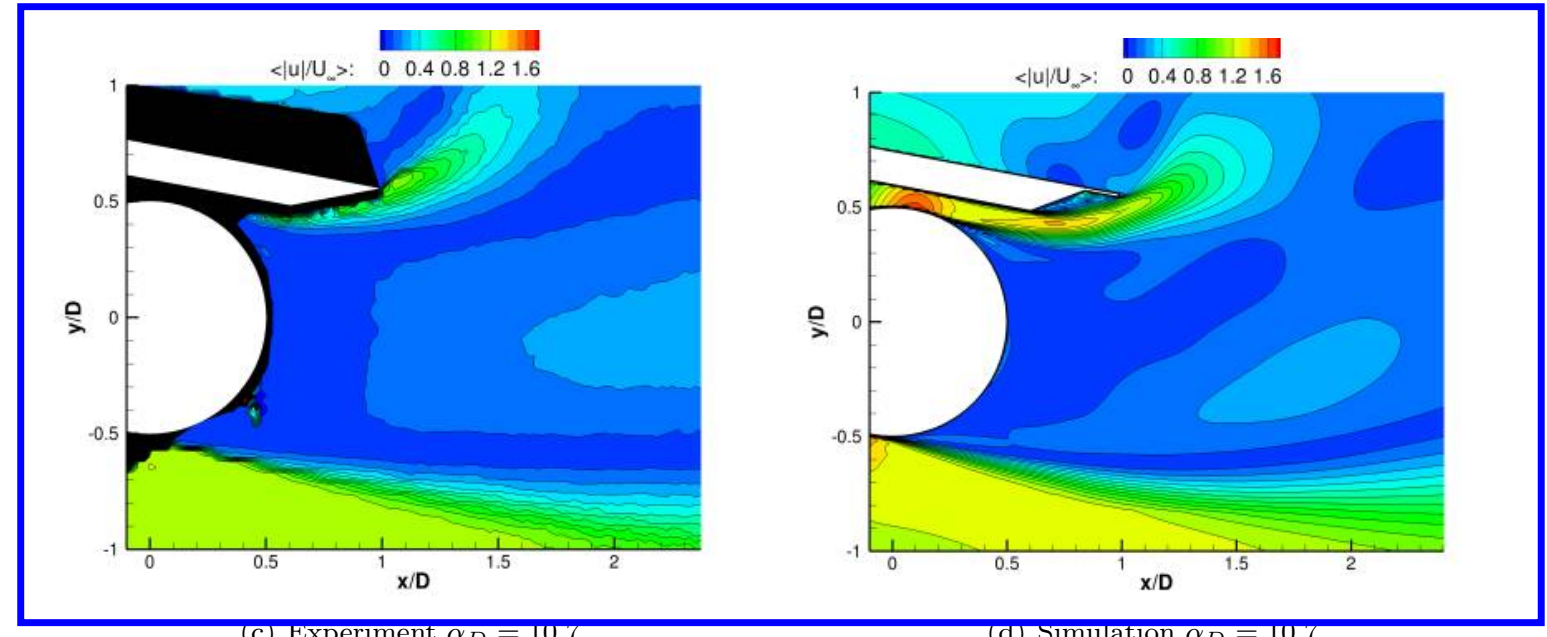

(c) Experiment $\alpha_{D}=10.7$

(d) Simulation $\alpha_{D}=10.7$

Figure 12. Comparison of the time averaged velocity magnitude field. 
features seem to be captured well by the 3-D simulation, however there seems to be a greater disagreement along $y / D>0.5$. This is thought to be attributed to the difference in the leg-door trailing edge models between the experiment and simulation, which can be noted within Figure 12. The model used in the experiment has a less discontinuous profile forming a thinner shear layer detaching towards the leg-door trailing edge. This causes the experimental turbulent intensity trends being consistently lower than the numerical simulations. The consistent over prediction by the simulations may also suggest that the eddy viscosity may be slightly over predicted, causing the turbulent wake to form into more coherent structures. Overall, the trends for varying door angle seems to be in agreement between the numerical and experimental cases.

\section{IV.C. Microphone data}

The changes to the wake intensity behind the cylinder element are highlighted in this section by the microphone data. The microphone is placed on the cylinder element at the mid-span at a position of $\theta=90^{\circ}$, positioned directed into the downstream region.

The microphone data was recorded at two free stream velocities to determine the velocity scaling of the data. The velocity scaling was tested by normalizing the pressure spectra by the free stream dynamic pressure and the result, shown in Figure 15, indicate that recorded signal contains primarily hydrodynamic pressures.

The changes to the spectra under varying configurations are shown by Figure 15 and outlines an over all reduction in the signal intensity. This corresponds to the observation from the PIV data that the turbulent intensity behind the cylinder is reduced with increasing door angle. The spectra for the isolated cylinder

flow has also been included in Figure 15. It shows that under interaction, the spectral peak shifts to lower frequencies in a more broadband manner, which has been noted in the 3-D simulations as well. 


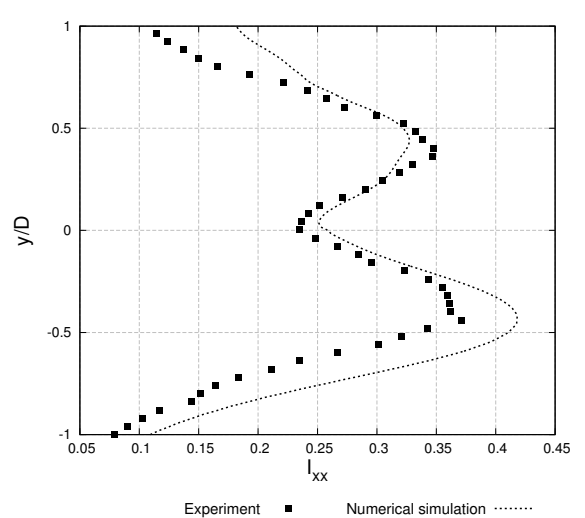

(a) $\alpha_{D}=0, x / D=1.5$

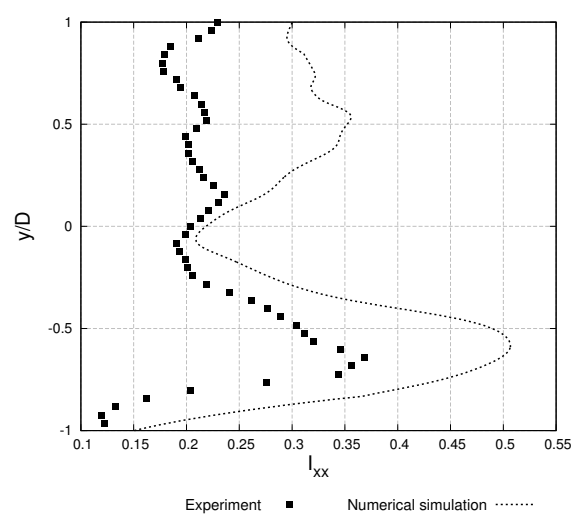

(c) $\alpha_{D}=5, x / D=1.5$

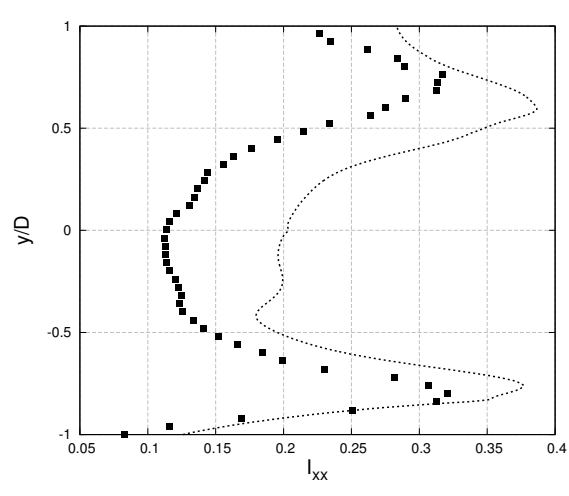

Experiment - Numerical simulation …......

(e) $\alpha_{D}=10.7, x / D=1.5$

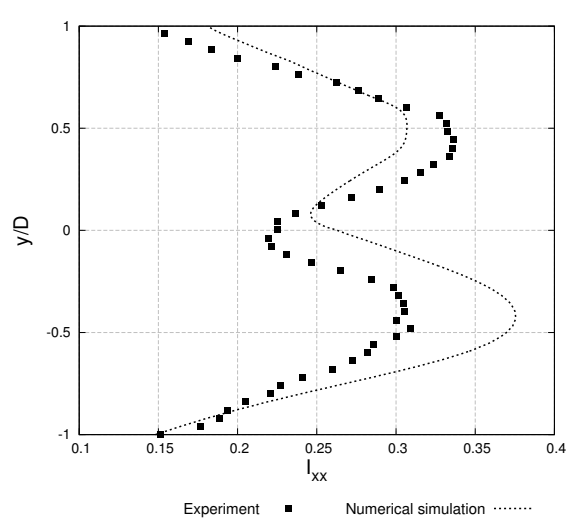

(b) $\alpha_{D}=0, x / D=2$

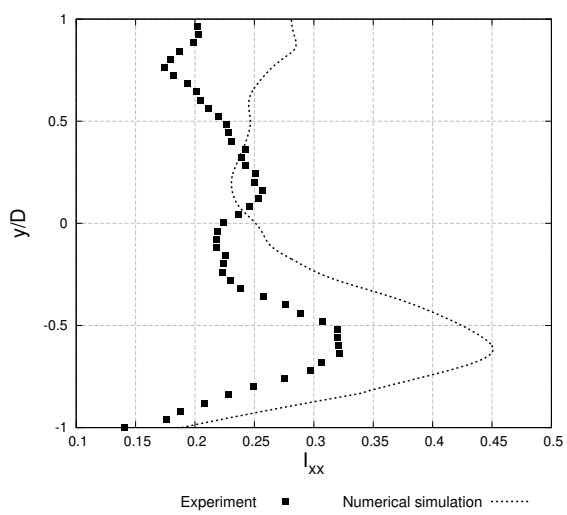

(d) $\alpha_{D}=5, x / D=2$

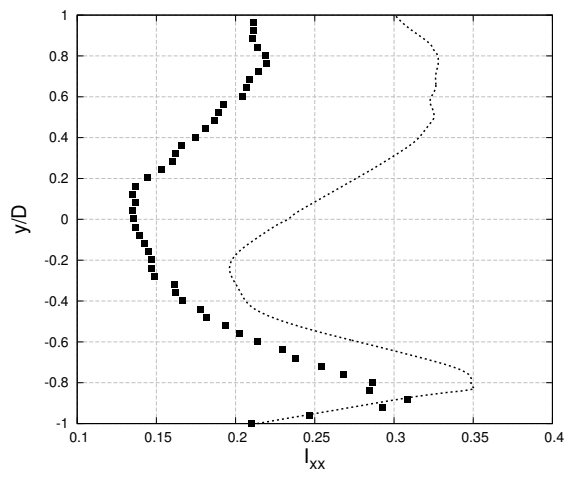

Experiment - Numerical simulation .........

(f) $\alpha_{D}=10.7, x / D=2$

Figure 13. Comparison of the non-dimensional turbulent intensity wake profile, based on $\overline{u / u \prime}$, between the simulation and experiment. 


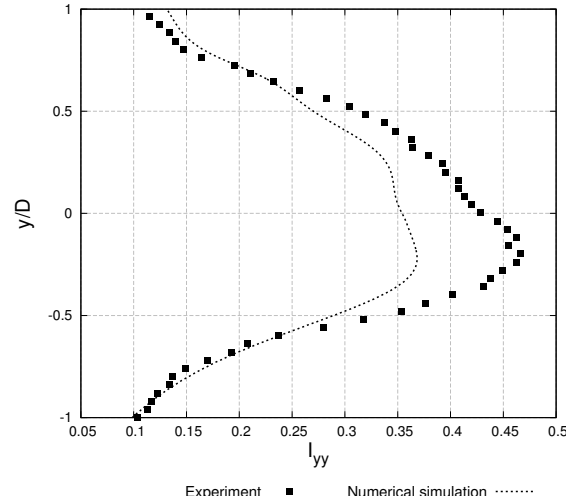

(a) $\alpha_{D}=0, x / D=1.5$

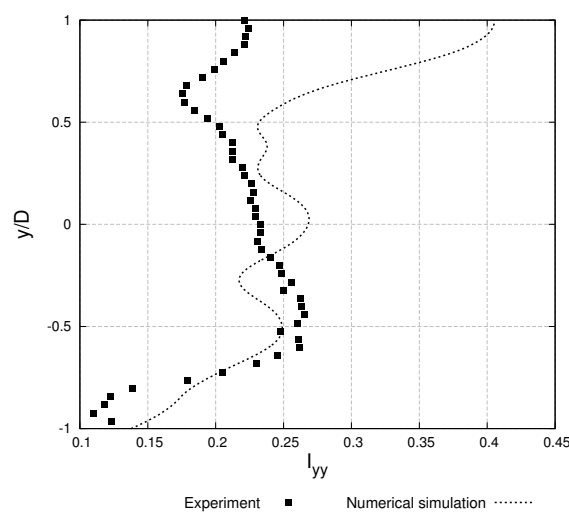

(c) $\alpha_{D}=5, x / D=1.5$

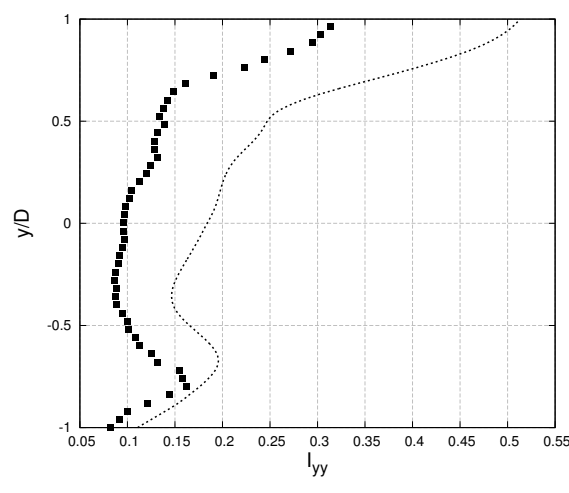

Numerical simulation........

(e) $\alpha_{D}=10.7, x / D=1.5$

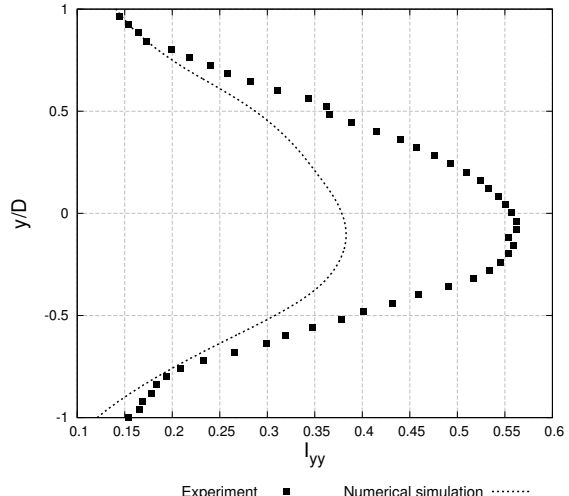

(b) $\alpha_{D}=0, x / D=2$

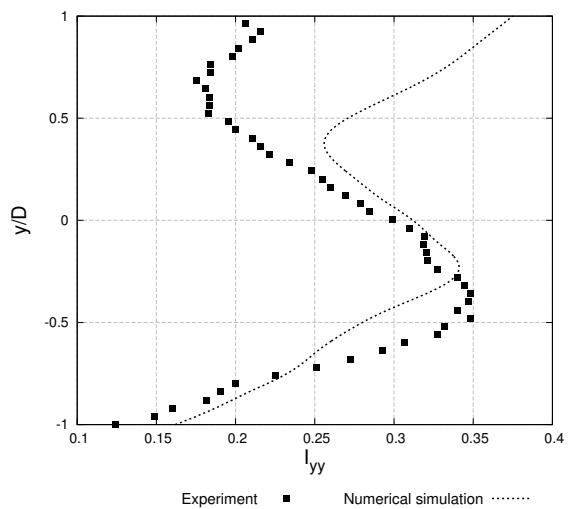

(d) $\alpha_{D}=5, x / D=2$

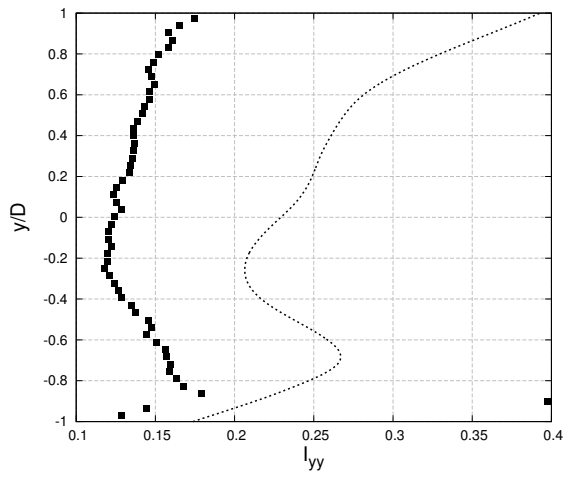

Experiment - Numerical simulation …......

(f) $\alpha_{D}=10.7, x / D=2$

Figure 14. Comparison of the non-dimensional turbulent intensity wake profile, based on $\overline{v / v \prime}$, between the simulation and experiment. 


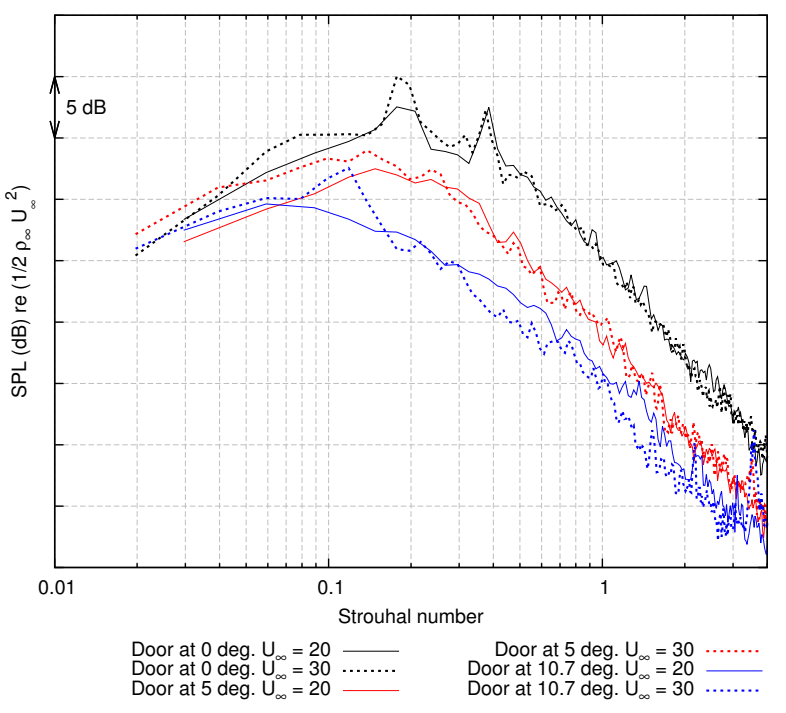

(a) Noise spectra scaling

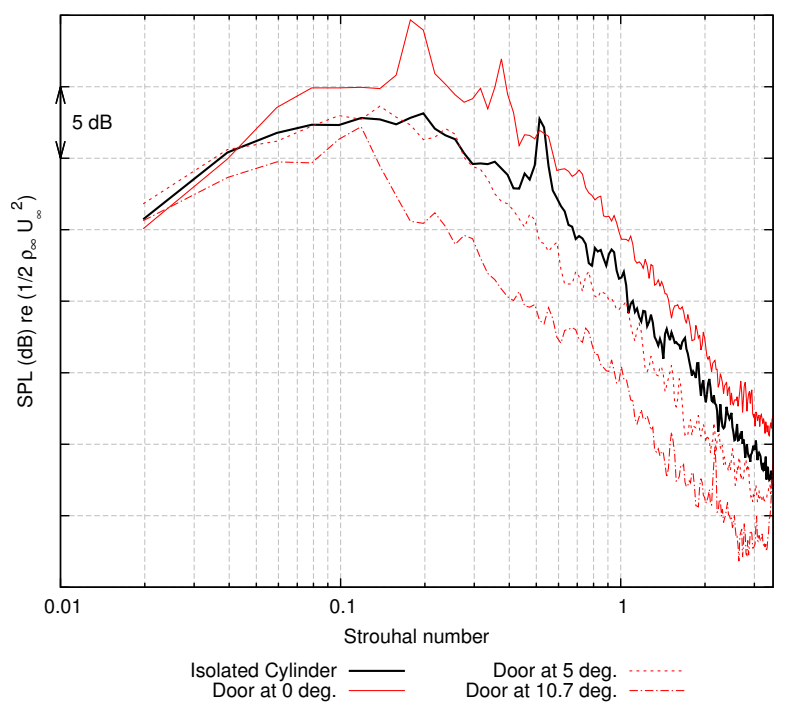

(b) Change in spectra due to interaction $U_{\infty}=30$

Figure 15. On surface microphone spectral data recorded at the mid-span of the cylinder element at $\theta=0^{\circ}$ for various interaction cases.

\section{Conclusion}

This paper studied the flow interaction between the leg-door and the main strut on an aircraft MLG. Two parameters, the door angle and the gap width ratio, were considered. A 2-D parametric study showed that increasing door angle has a similar effect as reducing the gap width in suppressing cylinder vortex shedding, which reduces the radiated sound from the main strut.

The flow physics and noise impact were further investigated through 3-D DDES simulations, using a compressible and higher order code on interaction cases at a gap width of $G=0.087$. Results showed that at low door angles a slight shadow region is formed for observers positioned on the door side of the model. This effect is not present when the door is placed at higher angles as the detached flow along the door surface becomes a more significant noise source. The OASPL increases by $10 \mathrm{~dB}$ from $\alpha_{D}=0$ to $\alpha_{D}=10.7$. A strong shear layer was identified at greater door angles, which drives a significant production in the mean turbulent kinetic energy in the wake region. This mechanism was associated to the interaction of shear layers from the separated flow on the upper surface of the door to the accelerated flow through the gap. The aeroacoustic data also shows that the far field spectra becomes more broadband with tones at lower frequencies, as the door angle is increased.

Experiments were conducted to determine the changes to the unsteady flow behind the cylinder element for a similar interaction model under the same door angle and gap settings. Microphone and PIV data show that the same trend, as captured by the 3-D simulations, exist. The door trailing edge was designed slightly differently to the simulation model for manufacturing purpose. Differences in turbulence intensity between measured data and simulations are believed to be caused by the simplified treatments at the door trailing edge. The current work has only investigated the interaction between a leg-door and the main strut model with an infinite span. Future work will investigate effect of the leg-door side edge and will be test various door angles.

\section{Acknowledgments}

The authors would like to thank Airbus for providing the funding and support of the project under which the current work is conducted. 


\section{References}

${ }^{1}$ Roshko, A., Steinolfson, A., and Chattoorgoon, V., "Flow Forces on a Cylinder Near a Wall or Near Another Cylinder," Proceedings of the 2nd U.S. National Conference on Wind Engineering Reserach, Colorado State University, Fort Collins, 1975.

${ }^{2}$ Bearman, P. W. and Zdrakovich, M. M., "Flow Around a Circular Cylinder Near a Plane Boundary," Journal of Fluid Mechanics, Vol. 89, No. 1, 1978, pp. 33-47.

${ }^{3}$ Nishino, T., Roberts, G. T., and Zhang, X., "Vortex Shedding From a Circular Cylinder Near a Moving Ground," Physics of Fluids, Vol. 19, No. 2, Article 025103, 2007.

${ }^{4}$ Nishino, T. and Roberts, G., "Absolute and convective instabilities of two-dimensional bluff body wakes in ground effect," European Journal of Mechanics - B/Fluids, Vol. 27, No. 5, 2008, pp. 539-551.

${ }^{5}$ Hutcheson, F. V. and Brooks, T. F., "Noise Radiation from Single and Multiple Rod Configurations," 12th AIAA/CEAS Aeroacoustics Conference, Cambridge, MA, AIAA Paper 2006-2629, 2006.

${ }^{6}$ Hedges, L. S., Travin, A. K., and Spalart, P. R., "Detached-Eddy Simulations Over a Simplified Landing Gear," Jounral of Fluids Engineering, Vol. 124, No. 2, 2002, pp. 413-423.

${ }^{7}$ Imamura, T., Hirai, T., Amemiya, K., Yokokawa, Y., Enomoto, S., and Yamamoto, K., "Aerodynamic and Aeroacoustic Simulations of a Two-wheel Landing Gear," IUTAM Symposium on Computational Aero-Acoustics for Aircraft Noise Prediction, Vol. 6, 2010, pp. 293-302.

${ }^{8} \mathrm{Li}$, F., Khorrami, M. R., and Malik, M. R., "Unsteady Simulations of a Landing-Gear Flow Field," 8th AIAA/CEAS Aeroacoustics Conference, Brekenridge, Colorado, AIAA Paper 2002-2411, June 2002.

${ }^{9}$ Lockard, D. P. and Khorrami, M. R., "Aeroacoustic Analysis of a Simplified Landing Gear," 10th AIAA/CEAS Aeroacoustics Conference, No. AIAA 2004-2887, 2004.

${ }^{10}$ Khorrami, M. R. and Lockard, D. P., "A Landing Gear Noise Reduction Study Based on Computational Simulations," INTER-NOISE, 2006.

${ }^{11}$ Humphreys, W. M. and Brooks, T. F., "Noise Spectra and Directivity for a Scale Model Landing Gear," International Journal of Aeroacoustics, Vol. 8, No. 5, 2009, pp. 409-443.

${ }^{12}$ Guo, Y., "A component-based model for aircraft landing gear noise prediction," Journal of Sound and Vibration, Vol. 312, No. 4-5, 2008, pp. 801-820.

${ }^{13}$ Lopez, L. V., "Prediction of Landing Gear Noise Reduction and Comparison to Measurements," 16th AIAA/CAES Aeroacoustics Conference, Stockholm, Sweden, AIAA Paper 2010-3970, June 2010.

${ }^{14}$ Cox, J. S., Brentner, K. S., and Rumsey, C. L., "Computation of Vortex Shedding and Radiated Sound for a Circular Cylinder: Subcritical to Transcritical Reynolds Number," Theoretical and Computational Fluid Dynamics, Vol. 12, No. 4, 1998, pp. 233-253.

${ }^{15}$ Orselli, R. M., Meneghini, J. R., and Saltara, F., "Two Dimensional Computation of Sound Generated by Flow Around a Circular Cylinder," 12th Brazilian Congress of Thermal Engineering Sciences, ABCM, Belo Horizonte, MG, November 2008.

${ }^{16}$ Farassat, F., "Derivation of Formulations 1 and 1A of Farassat," Tech. rep., National Aeronautics and Space Administration, March 2007.

${ }^{17}$ Spalart, P., Deck, S., Shur, M., Squires, K. D., Strelets, M. K., and Travin, A., "A New Version of Detached-Eddy Simulation, Resistant to Ambiguous Grid Densities," Theoretical and Computational Fluid Dynamics, Vol. 20, No. 3, 2006, pp. 181-195.

${ }_{18}$ Ashcroft, G. and Zhang, X., "Optimized Prefactored Compact Schemes," Journal of Computational Physics, Vol. 190, No. 2, 2003, pp. 459-479.

${ }^{19} \mathrm{Li}$, J., Li, F., and E, Q., "A Fully Implicit Method for Steady and Unsteady Viscous Flow Simulations," International Journal of Numerical Methods in Fluids, Vol. 43, No. 2, 2003, pp. 147-163.

${ }^{20}$ Peers, E., Zhang, X., and Kim, J. W., "Patched Characteristic Interface Conditions for the High-Order Multiblock Aeroacoustics Computation," AIAA Journal, Vol. 48, No. 11, 2010, pp. 2512-2522.

${ }^{21}$ Richards, S., Zhang, X., Chen, X., and Nelson, P., "The Evaluation of Non-Reflecting Boundary Conditions for Duct Acoustic Computation," Journal of Sound and Vibration, Vol. 270, No. 3, 2004, pp. 539-557.

${ }^{22}$ Wang, X. K. and Tan, S. K., "Comparison of Flow Patterns in the Near Wake of a Circular Cylinder and Square Cylinder Placed Near a Plane Wall," Ocean Engineering, Vol. 35, No. 5-6, 2008, pp. 458-472. 